\title{
Tangence
}

\section{L'autobiographie comme reprise : l'exemple d' Enfance de Sarraute}

\section{Barbara Havercroft}

Numéro 42, décembre 1993

Le récit de soi

URI : https://id.erudit.org/iderudit/025793ar

DOI : https://doi.org/10.7202/025793ar

Aller au sommaire du numéro

Éditeur(s)

Tangence

ISSN

0226-9554 (imprimé)

1710-0305 (numérique)

Découvrir la revue

Citer cet article

Havercroft, B. (1993). L'autobiographie comme reprise : l'exemple d' Enfance de Sarraute. Tangence, (42), 131-145. https://doi.org/10.7202/025793ar d'utilisation que vous pouvez consulter en ligne.

https://apropos.erudit.org/fr/usagers/politique-dutilisation/ 


\section{L'autobiographie comme reprise: l'exemple d'Enfance de Sarraute ${ }^{1}$}

\section{Barbara Havercroft}

Il n'y a pas de nouvelle matière, seulement une sorte de mémoire qui nous pousse vers l'avant. ${ }^{2}$

Les critères de l'autobiographie, comme ceux de tout genre d'ailleurs, n'ont cessé d'évoluer et de se renouveler, par un questionnement des limites et des contraintes de ses propres frontières. Si l'autobiographie et, en même temps, la critique de l'autobiographie ont connu un passage historique où l'accent a été mis d'abord sur le bios, ensuite sur l'autos, et enfin sur le graphe $^{3}$, il n'en demeure pas moins que la mémoire, le rôle des souvenirs et la répétition sont partie intégrante de tout texte autobiographique, quelle que soit sa date de parution. Ces constances de l'autobiographie donnent lieu à toute une chaîne syntagmatique associée à la description de cette pratique scripturale, où l'on constate la répétition du préfixe "re": l'autobiographie comme reconstruction ou reconstitution de l'histoire de la vie par l'écrit, la représentation de l'autobiographe et de sa personnalité, le retour en arrière requis pour ce réexamen de soi, sans oublier

1 Cet article fait partie d'un projet de recherche intitulé Les nouvelles formes de l'autobioqrapbie: théories et pratiques, qui est subventionné par le CRSH du Canada et l'Université du Québec à Montréal.

2 France Daigle, La beauté de l'affaire: fiction autobiographique à plusieurs voix sur son rapport tortueux au langage, Moncton/Montréal, Éditions d'Acadie/NBJ, 1991, p. 8.

3 C'est à partir de ce découpage étymologique que James Olney retrace I'histoire de l'entreprise autobiographique. D'après lui, les autobiographes et les critiques de ce genre manifestaient en premier lieu une préoccupation de la vie "réelle" de l'auteur(e) telle que représentée par le texte, en passant par l'enquête du "moi" (l'autos), pour aboutir aux études et aux pratiques actuelles axées surtout sur la textualité de l'écriture autobiographique. Voir James Olney, :Autobiography and the Cultural Moment: A Thematic, Historical, and Bibliographic Introduction", in J. Olney (éd.), Autobiograpby: Essays Theoretical and Critical, Princeton, Princeton University Press, 1980, p. 3-27. 
la dimension rétrospective du texte et la recréation poétique soulignées par Lejeune ${ }^{4}$. Malgré cette récolte abondante de vocables réitérant la reprise nécessaire à toute écriture autobiographique, ledit processus ne se réduit pas à une simple reproduction mimétique du hors-texte. Autant d'interférences - les trous de la mémoire, la fiction, le rêve, l'invention, les discours d'autrui, pour en nommer quelques-unes - viennent troubler la reprise autobiographique, de sorte que l'écriture autobiographique s'avère l'acte même de produire la différence par la répétition, où cette dernière n'est jamais une copie exacte d'une sensation ou d'un événement antérieurs. L'autobiographie, cette "entreprise impossible " 5 , se révèle plutôt comme le lieu du "développement du moi comme une négociation sans fin entre l'événement et l'illusion, le réel et l'imaginaire [...].6 ${ }^{6}$. Penser l'autobiographie en termes de répétition ou de reprise implique donc une reconsidération de ces derniers termes, un remaniement qui regrouperait aussi bien le même que l'autre, une (re)création nouvelle.

C'est à cette conception de la reprise, telle que l'expose Kierkegaard dans son texte du même titre ${ }^{7}$, à savoir en tant que renouvellement, un mouvement à la fois en arrière et en avant, que je m'attarderai dans ma relecture du texte de Sarraute. La reprise, texte publié le même jour que Crainte et tremblement, est en fait une autobiographie inavouée, où Kierkegaard essaie d'expliquer tant à Régine Olsen qu'à lui-même les raisons pour lesquelles il a rompu leurs fiançailles. Petit livre qui échappe à un classement générique spécifique, La reprise est un essai philosophique et éthico-religieux, de même qu'une œuvre de fiction qui contient un dialogue par correspondance écrite (des lettres fictives) et un discours sur le théâtre. Dans ce court texte attribué au pseudonyme de Constantin Constantius (où la reprise onomastique est loin d'être fortuite), le narrateur (Constantin) raconte l'histoire

4 Proposée dans sa célèbre définition de l'autobiographie, Le pacte autobiographique, Paris, Seuil, 1975 , p. 14. Philippe Lejeune a retravaillé cette définition dans "Le pacte autobiographique (bis)", Moi aussi, Paris, Seuil, 1986, p. 1334.

5 Philippe Lejeune, Moi aussi, op. cit., p. 34.

6 Janice Morgan, "Femmes et genres littéraires: le cas du roman autobiographique ", Protée, vol. XX, no 3, 1992, p. 28.

7 Sören Kierkegaard, La reprise, trad. Nelly Viallaneix, Paris, Flammarion, (1843) 1990. Toute référence au texte sera désormais indiquée par le folio entre parenthèses. 
de sa rencontre avec un jeune homme anonyme, follement amoureux, qui rompt, lui aussi, avec sa fiancée. Tout en donnant ses conseils au jeune malheureux, Constantin, à l'instar de Socrate, aide cet amoureux à explorer sa personnalité et son être, en particulier sa dimension poétique, ce qu'il ne peut réaliser qu'en perdant son amante. Pourtant, le jeune homme ne connait pas la vraie reprise, un renouvellement qui lui aurait permis de "retrouver l'exaltation du premier instant de l'amour [...] recréée à chaque aube nouvelle" 8 . Quant à Constantin, sa propre recherche de la reprise au cours d'une deuxième visite, d'un retour à Berlin, se termine, elle aussi, par un échec. Enfermé dans la nostalgie du souvenir, figé dans ses habitudes (un durcissement dans le "même", d'où la pertinence de son nom propre), Constantin le stöicien ne parvient qu'à la simple répétition, la reconstitution des circonstances antérieures, sans pour autant y ajouter la nouveauté. La structure même de ce texte énigmatique, une recréation soigneuse de reprises, d'échos et de refrains, voire un vaste jeu de variations sur certains thèmes dominants qui n'est pas sans rappeler une symphonie, constitue elle-même une parfaite reconstruction par sa forme de la problématique philosophique dont il s'agit ${ }^{9}$.

Le concept de reprise chez Kierkegaard est à comprendre de manière dialogique, où le sens ressort d'un contraste avec le ressouvenir:

Reprise et ressouvenir sont un même mouvement, mais en direction opposée; car ce dont on a ressouvenir, a été: c'est une reprise en arrière; alors que la reprise proprement dite est un ressouvenir en avant (p. 65).

Tandis que le ressouvenir s'oriente entièrement vers le passé afin de retrouver ce qui a été, la reprise vise également à retrouver le passé, mais sous une forme nouvelle et différente qui se rend vers l'avenir. Catégorie paradoxale, la reprise réaffirme simultanément "la similarité et la discontinuité " 10 et réunit en elle le même et l'autre.

8 Nelly Viallaneix, "Introduction", dans Sören Kierkegaard, op. cit., p. 18.

9 Parmi les nombreux exemples de ces reprises textuelles, on constate que le premier paragraphe de la deuxième (et dernière) partie du livre reprend celui de la première partie, ce demier paragraphe faisant également l'objet d'un réexamen dans un autre passage, toujours dans la deuxième partie, où Constantin rappelle le concept de ressouvenir chez les Grecs dans ses commentaires sur les lettres du jeune homme innommé (p. 130).

10 Ce sont les mots de Michael Sprinker, dans son article "Fictions of the Self: The End of Autobiography ", dans J. Olney (éd.), op. cit., p. 329. Sprinker relie 
Selon Kierkegaard, la reprise doit être saisie en termes de médiation (non pas pour aboutir à l'Aufbebung hégelienne, à l'égard de laquelle il était fort critique), conçue comme un mouvement de

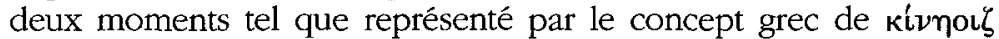
(passage, transition, motion, changement). Ainsi la dialectique de la reprise est-elle décrite de la manière suivante: "ce qui est re-pris, a été, sinon, il ne pourrait pas être re-pris; mais, précisément, c'est le fait d'avoir été qui fait de la re-prise une chose nouvelle" (p. 87). À la différence du simple ressouvenir, la vraie reprise implique donc un renouvellement, du renouveau. Processus créateur, l'acte de retrouver le même doit nécessairement s'effectuer d'une façon inédite.

La notion de mouvement ou de passage évoquée ci-haut est inhérente à la reprise kierkegaardienne, qui se révèle être, en fin de compte, une catégorie religieuse chrétienne. La reprise comme parcours individuel intérieur à travers la vie comprend plusieurs étapes différentes, des "saut[s] qualitatif[s] pour passer d'un ordre à un autre situé plus haut "11. De ces divers stades, Kierkegaard en distingue trois, dont chacun est incarné par un représentant spécifique: l'étape érotique et esthétique (le jeune amoureux devenu poète), l'étape éthique (Constantin), et l'étape religieuse (incarnée par Job, qui devient une sorte de modèle pour le jeune homme après sa rupture). Il ne s'agit pas d'abandonner une étape dès que l'on arrive à la suivante: au contraire, chaque phase retient sa valeur à tout moment, et la nouvelle étape offre une reconsidération de la dernière. Le but ultime de ce parcours, que n'atteignent ni le jeune homme ni Constantin, c'est la renaissance chrétienne, le recommencement de la vie en tant que recréation spirituelle. Ce dernier stade éthico-religieux fait l'objet d'une analyse approfondie dans Crainte et tremblement, tandis que $L a$ reprise est plutôt consacrée aux deux côtés de l'étape esthétique: l'eros, l'amour-désir et l'amour-passion (le domaine érotique) et l'esthète comme juge de la beauté et de l'art (d'où les nombreux commentaires traitant du théâtre) ${ }^{12}$.

la reprise kierkegaardienne à la conception que propose Vico de l'histoire en tant que répétition ou récurrence avec différence.

11 Nelly Viallaneix, op. cit., p. 19.

12 Mon analyse d'Enfance ne tiendra pas compte de cet aspect religieux de la reprise kierkegaardienne, puisque Sarraute n'envisage pas de renouvellement de ce genre au cours de son réexamen autobiographique. Je me servirai uniquement des autres dimensions de la reprise, telles qu'elles ont été élaborées ci-haut, qui sont plus pertinentes à l'étude du texte de Sarraute. 
Dans Enfance, Sarraute reconstruit et réexamine les événements, les personnes, et avant tout, les paroles et les sensations qui ont marqué sa vie, de l'âge de deux à douze ans ${ }^{13}$. Utilisant une série de 70 fragments dont la structure n'est pas sans rappeler celle de Tropismes ${ }^{14}$, Sarraute représente les épisodes significatifs de son enfance, ces déclencheurs des émotions ressenties au temps de l'énoncé aussi bien qu'au moment del'énonciation autobiographique, c'est-à-dire lors de leur inscription et de leur réinterprétation. Ce qui importe, c'est moins les paroles et les événements répétés que leur capacité de faire revivre les sentiments d'autrefois, de relancer le tourbillonnement des tropismes intérieurs et, à l'occasion qu'offre cette reprise autobiographique, de les réanalyser. Ayant été renvoyée sans cesse entre deux parents séparés (et ensuite, divorcés), entre deux cultures, entre deux pays (la Russie et la France) et par conséquent, entre deux langues et deux noms propres (Natacha et Nathalie), Sarraute reproduit ce va-et-vient constant de sa jeunesse par le dialogue et par le débat des deux voix énonciatives qui racontent et commentent son autobiographie et sa (re)mise en texte ${ }^{15}$. Parmi les nombreuses formes de reprise parsemées dans le texte de Sarraute, il y en a quatre qui retiendront mon attention dans cette brève étude: la réénonciation effectuée par les deux voix narratives, l'usage récurrent de nombreux lexèmes ayant tous le préfixe "re", la reprise d'épisodes qui se ressemblent aux niveaux du contenu et de la forme, et les reprises intertextuelles, où le texte autobiographique reprend certains éléments de l'œuvre littéraire de l'écrivaine.

Comment effectuer un renouvellement qui entraînerait un mouvement à la fois en arrière et en avant? Comment s'y prendre pour retrouver son passé tout en s'orientant vers l'avenir? Pour

13 Que cette nouvelle romancière ait pu publier un texte centré sur la recherche de soi n'a pas manqué d'étonner certains critiques. D’autres, par contre, ne voient aucune rupture avec son ouvre antérieure. À cet égard, voir Bruno Vercier, "(Nouveau) Roman et autobiographie: Enfance de Nathalie Sarraute ", French Literature Series, vol. XII, 1985, p. 162-170.

14 Nathalie Sarraute, Tropismes, Paris, Denoël, 1939; réédition, Paris, Minuit, 1957.

15 En dépit du caractère discontinu du texte et de la rareté de repères temporels, il est toutefois possible de reconstruire les dates et les lieux de cette enfance selon un ordre chronologique rigoureux, comme l'a fait Philippe Lejeune dans "Paroles d'enfance" Revue des sciences bumaines, vol. XCIII, $\mathrm{n}^{\circ} 217$, janvier-mars 1990 , p. $25-26$. 
Kierkegaard, la reprise ainsi conçue exige l'intersubjectivité et le dialogue: c'est à travers l'autre, ou soi-même divisé et postulé comme un autre, que la similarité et la discontinuité nécessaires à la vraie reprise se produisent. Tout en se cachant derrière son pseudonyme, Kierkegaard fait parler son autre fictif, Constantin Constantius, qui, à son tour, fait discourir le jeune homme amoureux. Non seulement Kierkegaard est-il incarné dans ses deux personnages ${ }^{16}$, mais ces deux "autres" insistent sur l'exigence de parler avec l'autre, quel qu'il soit, afin de parler avec soi-même ${ }^{17}$. Qui plus est, les discussions de Constantin avec cet amoureux et, ensuite, les lettres qu'écrit ce dernier à son confident permettent un réexamen de soi de la part de ces deux protagonistes. Même si cette construction enchâssée de personnages et de paroles rappelle celle de la ventriloquie, les relations entre les énonciateurs interdépendants ne se réduisent pas à une hiérarchie de marionnettes, manipulées par un autre, situé dans un hors-texte. Les paroles et les destins de Constantin et du jeune homme s'informent mutuellement, car chacun répète et reformule les mots et les silences de l'autre. De cette façon, le texte de $L a$ reprise, ainsi que l'expérience de la reprise elle-même, émanent d'une "intersubjectivité où deux personnages inventés se parlent en répétant ce que l'autre n'a pas dit " ${ }^{18}$.

Il en va de même dans le texte de Sarraute, où le "je" autobiographique (déjà dédoublé, comme dans tout écrit autobiographique, entre le "je" de l'énoncé et celui de l'énonciation) se scinde pour instaurer un dialogue entre deux voix énonciatives qui mettent en question l'unicité du sujet autobiographique, son autorité vis-à-vis de son discours, de même que l'acte de reproduire le souvenir. Ainsi, l'ère du soupçon ${ }^{19}$ se répand-elle du domaine romanesque pour envahir celui du récit de soi, de sorte

16 D’après Viallaneix, Kierkegaard "se dédouble: tandis qu'il investit dans le personnage de Constantin la partie "raisonnable" de son être, il attribue l'autre, la "sentimentale", au jeune homme", d'où l'emploi du pseudonyme et du vide onomastique. Voir Nelly Viallaneix, "Introduction", op. cit., p. 15.

17 Constantin signale cette urgence chez le jeune homme qui "avait besoin d'un confident, en présence duquel il pût parler tout haut avec lui-même" (p. 70). Dans une de ses lettres à Constantin, l'homme innommé avoue: "Parler avec vous me procure un indescriptible et bienfaisant soulagement; c'est comme parler avec soi-même ou avec une idée» (p. 132).

18 Ce sont les mots de Michael Sprinker, op. cit. (ma traduction).

19 Voir Nathalie Sarraute, L'ère du soupçon, Paris, Gallimard, 1956. 
que la reprise entraîne une (re)négociation intersubjective où le passé ressurgit dans le présent de l'écriture, tandis que le présent se replonge dans le passé pour le réactualiser et le réinterpréter, même si la reprise des événements et des sensations n'est qu'approximative. Si l'une des deux voix s'approprie le rôle de la narratrice et l'autre celui du commentateur ${ }^{20}$, cette attribution de fonctions énonciatives n'est pas tout à fait figée, puisque la mobilité des identités est annoncée vers le début du récit ${ }^{21}$. Malgré ce flottement, la voix narratrice est en général consacrée à la représentation de l'histoire de l'enfance, tandis que la voix critique assume d'autres fonctions, en l'occurrence, pour reprendre l'analyse de Lejeune ${ }^{22}$, le contrôle ("l'exercice du soupçon" et la remise en question de l'exactitude de la narration), l'écoute (une interrogation sur le sens des éléments du passé revisité), et la collaboration avec la voix narratrice. Il importe de noter que cette division binaire de voix est nettement plus complexe dans son enchevêtrement du passé et du présent, du souvenir (parfois flou) et du commentaire que ces quelques remarques le laissent croire. Par moments, l'enchâssement des énonciations prend des dimensions étonnantes, comme, par exemple, dans le passage où la voix narratrice se souvient de Natacha (à neuf ans) en se souvenant de ce qu'elle a vécu un an auparavant ${ }^{23}$. En outre, ce chapitre recourt à des verbes dénotant simultanément la reprise et la difficulté d'un tel processus: retenir, retrouver, revoir, revenir, repenser, remettre, et ainsi de suite.

20 D’où la remarque de Lejeune, qui les caractérise en tant que "voix narratrice" et "voix critique" dans "Paroles d'enfance", op. cit., p. 32-33. Il importe de noter les adjectifs au masculin attribués à la voix critique au début du texte (p. 8-9), d'où mon emploi du substantif "commentateur".

2l Comme le note avec raison Lejeune, la voix narratrice "se révolte contre la voix critique en l'accusant à son tour de replâtrage". Voir Philippe Lejeune, op. cit., p. 33. Puisque les études critiques d'Enfance se sont longuement arrêtées sur cette structure dialogique, c'est la pertinence de celle-ci en ce qui concerne la mise en texte de la reprise qui fera l'objet de mes propres commentaires. Parmi les études des voix énonciatives dans Enfance, on peut consulter Françoise Van Roey-Roux, "Enfance de Nathalie Sarraute ou de la fiction à l'autobiographie", Études littéraires, vol. XVII, no 2, 1984, p. 273282, et Raylene O'Callaghan, "Voice(s) in Nathalie Sarraute's Enfance", New Zealand Journal of French Studies, vol. IX, n 1, 1988, p. 83-94.

22 Philippe Lejeune, "Paroles d'enfance", op. cit., p. 34-35.

23 Nathalie Sarraute, Enfance, Paris, Gallimard, coll. "Folio *, 1983, p. 91-103. Toute référence à ce texte sera désormais indiquée par le folio entre parenthèses. 
138

Afin d'illustrer l'impossibilité, voire la futilité du ressouvenir exact, les deux voix se combinent pour retravailler un énoncé ou une scène spécifique. Tel est le cas de l'énoncé, répété par la voix narratrice, que profère la mère de Nathalie à sa fille ("Un enfant qui aime sa mère trouve que personne n'est plus beau qu'elle", p. 95) suite à une remarque franche de la part de la jeune fille: "[Maman] Je trouve qu'elle [une poupée] est plus belle que toi" (p. 95). Ne retenant que des paroles blessantes - les actions suivant cet échange ayant disparues dans le brouillard des modalisateurs multiples ("peut-être nous taisions-nous ou peut-être même avons-nous continué à parler", p. 95) - , les deux voix discutent des interprétations possibles de l'énoncé de la mère. S'ensuit toute une série de reformulations plus acceptables de la phrase maternelle ${ }^{24}$, en fonction de l'écart temporel, mais en retenant la douleur ressentie par la fille. Après une première conclusion de condamnation de soi, la narratrice fait " réapparaître devant [elle] [le] visage rose, lumineux [de la mère]" (p. 97) pour la réexaminer, et finit par se détacher d'une conception stéréotypée "[d]es autres, [d]es vrais enfants" (p. 98) à laquelle elle ne semble pas adhérer. Ce réexamen déclenche chez elle les débuts d'un processus de réaffirmation de soi, la croyance qu'elle a droit à ses propres opinions, qu'elle n'est peut-être pas si méchante. Après avoir péniblement reconstruit d'autres épisodes et d'autres paroles qui remettent en question aussi bien l'amour de la fille pour sa mère que le caractère autrefois irréprochable de celle-ci, la narratrice se lance vers l'avenir. Elle accepte que de telles "idées" et de tels doutes déconcertants vont inévitablement "revenir", qu'ils étaient prêts "à [re]bondir au cours de n'importe quel repas" (p. 103).

L'orientation vers l'avenir exprimée dans ce dernier énoncé s'avère une prévision exacte, d'ailleurs, qui vaut aussi bien pour les événements de la vie que pour l'écriture autobiographique. Voilà, justement, ce qui "revient" dans le fragment 36 (p. 135). C'est une reprise, mot pour mot, d'un des énoncés exprimant des "idées" inquiétantes de Nathalie au sujet de sa mère: "Tu sais, maman, j'ai mes idées... Je pense que tu as la peau d'un singe" (p. 135). Cette répétition énonciative (l'injure a été d'abord lancée lors du fragment concernant la poupée discuté ci-haut), qui re-

24 "Ce qu'elle avait sans doute voulu dire était: Un enfant qui aime sa mère ne la compare jamais à personne. - C'est ça: un enfant qui aime sa mère ne l'observe pas, il ne songe pas à la juger (p. 96). 
prend avec elle le poids affectif du premier contexte, permet à la narratrice de se réaffirmer pleinement, cette fois pour de bon. Insistant sur son droit à s'exprimer, sans crainte ni honte, la narratrice réénonce, avec plus de confiance, son indépendance:

Aucune [de ses "idées "] ne peut me faire honte, aucune ne peut m'atteindre. Oh que je me sens bien [...]. Il me semble qu'à ce moment-là, j’ai cru posséder pour toujours une force que rien ne pourrait réduire, une complète et définitive indépendance" (p. 136-137).

La reprise énonciative mène, dans cet exemple, à une (re)prise de conscience d'elle-même.

De cette manière, certaines scènes, même si leur reprise et leur "traduction" scripturale manquent de contours nets et solides, se prêtent toujours à une réanalyse. À cet égard, l'épisode raconté au fragment 17 est exemplaire. Ici, la voix critique aide la voix narratrice à faire ressurgir un souvenir douloureux (le rejet de Nathalie par sa mère et Kolia) et ensuite à réinterpréter la scène trois fois de suite. Chaque possibilité devient de plus en plus angoissante, allant d'un simple sentiment d'écart (comparé au "tintement d'un verre doucement cogné ", p. 74) à un dérangement ("je dérangeais leur jeu", p. 75), pour aboutir à une représentation de soi en tant que "corps étranger [...] qui gênait" (p. 75). Pourtant, la réinterprétation de quelques scènes et énoncés se limite parfois aux seules retouches, effectuées à de simples détails. Les deux voix collaborent, par exemple, pour refaire la description des façades des maisons à Pétersbourg ("mornes... - Mortes, devrais-tu dire... - Oui, sans vie", p. 113) et pour remanier la qualification d'un pressentiment: "Plus" ne convient pas. "Autre" serait mieux" (p. 166).

Si le seul choix des mots se prête à un remaniement autobiographique englobant le passé, le présent, et l'avenir, l'emploi ré$\mathrm{Cu}^{+}$ient des verbes comportant le préfixe "re" le rend encore plus évident. Ces nombreux vocables servent non seulement à véhiculer le processus de la reprise (que ces lexèmes soient, pour la plupart, des verbes, accentue d'autant plus le mouvement inhérent à la reprise), mais également à attirer l'attention à cette remémoration. La répétition de tels verbes ${ }^{25}$ dans maints contextes

25 Au lieu de fournit une liste exhaustive de tous ces verbes, je me contenterai des exemples suivants: revenir, retenir, retrouver, reconnaitre, revivie, revoir, 
différents met en évidence le mouvement (en arrière et en avant) de la reprise, la revitalisation du passé dans le présent de l'énonciation autobiographique, ce qui amène les deux voix à une reconstitution constante du sujet autobiographique et de son histoire. L'emploi du présent comme temps du verbe appliqué aux événements du passé ne fait que réactualiser ceux-ci, en donnant "l'impression d'une redécouverte que le narrateur fait lui-même [sic] au moment de la narration" 26 . Enfin, et cette fonction métatextuelle est loin d'être la moins importante de cette remarquable récurrence lexicale, les deux voix énonciatives se servent parfois de ces verbes de reprise pour représenter ou pour commenter l'acte même de (re)créer une reprise autobiographique.

Dans le fragment 47 , par exemple, c'est le verbe "reprendre" lui-même qui instaure tout un échelonnement de (re)présentations qui confondent le passé, l'avenir de l'enfant (pas encore raconté) et le présent de la narration autobiographique. Malgré "le brouillard qui [...] recouvre [le passé]"(p. 172), la voix narratrice raconte l'épisode où la mère suggère qu'elle reprenne sa fille, que la petite revienne habiter chez elle, pourvu que le père se charge de la renvoyer. La reconstruction de cette scène provoque la remémoration d'une autre, car la voix narratrice se souvient d'elle-même en se souvenant de la douleur déjà ressentie au cours de la séparation d'avec la mère, "cette brusque réapparition de ce à quoi j'avais été arrachée, que je m’étais efforcée d'écarter" (p. 173). Ayant en partie oublié sa vie antérieure en Russie chez sa mère pour pouvoir se faire une place chez son père et Véra à Paris, la narratrice se revoit, fâce à cette décision difficile, et en revit, même au moment de la narration autobiographique, le poids affectif: "Quelque chose s'élève encore, toujours aussi réel, une masse immense...cela me redresse, me soutient, me durcit" (p. 173, c'est moi qui souligne). Dans ce passage, l'emploi du présent et des adverbes "encore" et "toujours" peuvent s'appliquer aussi bien au temps de l'énoncé (quand la

relire, ressusciter, reconstituer, repenser, réentendre, ressortir, remettre, recommencer, ressentir, réapparaittre, reproduire.

26 Françoise Van Roey-Roux, "Enfance de Nathalie Sarraute ou de la fiction à l'autobiographie ", op. cit., p. 276. Comme le signale Van Roey-Roux avec justesse, le présent fonctionne également pour réaccentuer l'émotion ressentie au cours de l'événement raconté, pour rendre "plus plausible l'intensité de l'émotion". 
petite s'est décidée de rester chez son père) qu'au temps de l'énonciation, au moment de la reprise. En outre, le processus de repenser et de réexprimer sa réaction défensive, provoquée par "ce brutal rapprochement [qui mènerait peut-être à] la découverte d'un nouvel éloignement" (p. 173), est lui-même remis en question, la voix narratrice ayant toujours peur que son écriture et ses souvenirs russes ne se réduisent à de simples clichés:

Mais cette reconstruction de ce que j'ai dû éprouver est pareille à une maquette en carton reproduisant en un modèle réduit ce qu'avaient pu être les bâtiments, les maisons, les temples, les rues, les places et les jardins d'une ville engloutie (p. 173).

Dans ce fragment, l'acte de reconstituer une reprise littérale (le possible retour de Nathalie en Russie), facilitée et véhiculée en partie par des lexèmes comportant le préfixe "re" (qui mettent en évidence cette reconstruction d'une reprise de l'enfant), rend également possible un réexamen à la fois de soi et de l'écriture.

La reprise de certaines scènes spécifiques dans Enfance constitue encore un autre procédé textuel qui contribue à l'expérience de la reprise dans le sens kierkegaardien du terme. En fait, pour reformuler ma propre idée de manière plus juste, ill serait plus exact de parler de scènes qui reprennent le même thème, les mêmes activités ou les mêmes émotions, comme il n'est pas question de "revisiter" le même événement en tant que tel ${ }^{27}$. Par cette "mise en relation d'éléments contigus" 28 , séparés par des écarts temporels et textuels, les différences d'attitude et de comportement chez l'enfant sont accentuées, ce qui nous permet, d'après Vercier, de suivre le parcours de l'enfant vers son indépendance "par rapport aux caprices et exigences de la mère" 29. Certes, ces répétitions textuelles mettent en relief ses réactions différentes dans des circonstances identiques, mais leur fonction n'est pas limitée à une simple comparaison de ressouvenirs. Si

27 Vercier range les épisodes suivants dans cette catégorie de "scènes reprises": les deux visites à l'usine du père (d'abord à Ivanovo [p. 50-52], ensuite à Vanves [p. 268-271]; les deux maladies différentes (où Nathalie est soignée la première fois par sa mère [p. 37-40] et la deuxième fois par son père [p. 223225]); les deux récitations (à la maison, pour les adultes [p. 62-63] et à l'école [p. 180-181]); et les deux épisodes de peur nocturne (chez la mère [p. 89-90] et chez le père [p. 245-246]).

28 C'est ainsi que Lejeune décrit le fonctionnement de cette reprise textuelle dans "Paroles d'enfance", op. cit., p. 30. 
ces épisodes se font écho, se répondent, impliquant de manière dialogique le même et l'autre, il n'en reste pas moins qu'ils s'orientent vers l'avenir (à travers le passé et le présent) du sujet autobiographique, aux niveaux du réexamen de soi et de l'écriture.

Cette problématique relative à la fois à la construction du sujet aussi bien qu'à l'écriture du texte autobiographique est à remarquer avant tout dans les deux scènes de rédaction. La première, où la voix narratrice revit l'angoisse de la petite Natacha lors de la condamnation de son roman (à cause de l'orthographe) par un ami de sa mère, reprend tous les éléments scripturaux auxquels Sarraute l'écrivaine s'est toujours opposée. Reconstituer "ces instants" de rejet pénibles, c'est ici reconnaître le caratère faux et figé des mot empruntés, "rigides et lisses, glacés" (p. 88), des mots stéréotypés et des personnages des contes de fée qui ne sont pas les siens. Repensé de cette façon, l'échec se transforme plutôt en "délivrance", puisque la voix narratrice (et la lectrice) est capable de reconnaître le contraste entre son écriture toute faite et stérile d'autrefois et implicitement, celle, vivante, des textes de Sarraute écrivaine.

La reconstitution de ce premier épisode sur la rédaction trouve son écho dans le fragment 55 , où les deux voix réexaminent le devoir de français intitulé "mon premier chagrin". Reprenant les problèmes de l'écriture, ce fragment autobiographique porte donc sur l'écriture antérieure d'un texte autobiographique - un bel enchâssement qui fait ressortir le contraste entre ces deux expériences scripturales. Incapable à l'époque de parler d'elle-même, la jeune Nathalie cherche "un chagrin qui serait hors de [s]a propre vie» (p. 208), réutilisant des citations et des expressions retrouvées dans des livres et des dictées. Empruntant ainsi un chagrin fictif (la mort d'un chien) ainsi que des mots ("comme revêtus de beaux vêtements") qui ne sont pas les siens, la fille produit un texte qui lui plaît, tellement il est " tout lisse et net et rond" (p. 214). Ce texte "immuable" (p. 214), écrit pour une autre (le professeur) sur le chagrin d'un "autre" fictif, s'oppose, encore une fois, et de façon remarquable, aux livres publiés par Sarraute, caractérisés par la fluctuation constante des mouvements intérieurs. Et c'est justement à l'occasion de la reprise autobiographique que constitue Enfance dans son entier, et l'épisode de la rédaction, en particulier, qui fait ressortir ce contraste. Incitée par l'exhortation persistante de la voix critique, la voix narratrice 
constate la nature stérile de ses premières tentatives scripturales et de sa propre peur "de voir quoi que ce soit se mettre à fluctuer, devenir instable, incertain" (p. 215). Cet réexamen lui permet de revoir le devoir de français pour ce qu'il était, ce qu'il représente toujours, ainsi que sa propre hésitation, jadis, de "dépasser les limites qui [lui] sont assignées" (p. 216).

En dernier lieu, signalons certaines reprises formelles et intertextuelles dans Enfance, ces nombreuses ressemblances aux textes déjà publiés par Sarraute. Que l'on commence par les caractéristiques générales (la séquence des scènes centrées surtout sur un énoncé spécifique ${ }^{30}$, le dévoilement de la sousconversation, la structure souvent hésitante des phrases, l'emploi des modalisateurs et les points de suspension, les métaphores liquides), ou que l'on s'attarde aux réemplois intertextuels spécifiques, on remarque que ce texte de Sarraute est une vaste construction textuelle de reprises, renouvelé par le versant autobiographique. Certains de ces derniers emprunts reprennent, même au niveau du vocabulaire, des passages déjà lus et vus. Tel est le cas du premier épisode de peur nocturne dans Enfance, marqué par un tableau effrayant qui met en scène des arbres menaçants: "c'est elle, cette allée d'arbres pointus, rigides et sombres [...] cette procession de fantômes revêtus de longues robes blanches" (p. 89). Cette description rappelle celle d'une autre peur nocturne, elle aussi suscitée par un tableau sur le mur qu'il faut couvrir pour que l'enfant se calme: "couvrez ce tableau, là [...] des sapins couverts de neige... des bras de fantômes se tendent" 31 . Dans les deux cas, la peur s'exprime avec l'intervention d'un autre; toutefois, ce qui a été révélé au niveau de la sousconversation dans Entre la vie et la mort se manifeste plus ouvertement dans Enfance, et de façon plus détaillée, dans le dialogue des deux voix. Tout comme en témoignent les autres exemples de reprises intertextuelles, il semble que les brèves descriptions amorcées auparavant trouvent leur pleine expansion au cours de leur réinscription autobiographique. L'enfant qui a ses "idées", évoqué brièvement par rapport aux "mots" dans Entre la vie et la

30 À cet égard, consulter Nathalie Sarraute, L'usage de la parole, Paris, Gallimard, 1980. Dans ce texte, chaque section commence par un énoncé en forme de titre qui fait ensuite l'objet d'une étude minutieuse.

31 Nathalie Sarraute, Entre la vie et la mort, Paris, Gallimard, coll. "Folio", 1968, p. 24. Cet exemple, de même que les autres mentionnés ci-haut, sont évoqués par Bruno Vercier, op. cit., p. 168. 
144

mort $^{32}$, revient, comme je l'ai montré dans mon étude du fragment 22 (p. 91-103), pour constituer le (pré)texte de tout un réexamen de soi et du processus de son devenir dans et avec le temps. Il en va de même pour les maisons russes, porteuses solides de connotations figées des contes de fée, par lesquelles s'ouvre Le silence ${ }^{33}$ et dont la portée se retrouve dans Enfance. Et si le caractère rigide du célèbre devoir sur le premier chagrin, de même que l'obéissance aveugle au modèle scolaire aux fins de l'approbation du professeur, font l'objet d'une courte discussion dans Entre la vie et la mort ${ }^{34}$, ce n'est que dans Enfance, lors de leur reprise, que leur signification est entièrement développée. Ces passages réutilisés ne sont pas que de simples ressouvenirs; ils deviennent, une fois repris dans Enfance, de vraies reprises, où l'ancien trouve son vrai épanouissement, un élargissement et un éclaircissement de son sens, au sein de son nouveau contexte.

Relu en fonction de la reprise kierkegaardienne, le texte autobiographique de Sarraute représente l'acte de produire la différence tout en reprenant le même. Grâce à de nombreux procédés textuels, Sarraute réussit à effectuer une reprise autobiographique qui incorpore le même mouvement et le même renouvellement que le texte fait ressortir au niveau métatextuel. Réaliser la reprise telle que conçue par Kierkegaard, c'est lutter contre la reproduction du même, contre le durcissement des habitudes. Cette lutte se reproduit dans Enfance tant au niveau de la reconstitution renouvelée des événements et des émotions du passé qu'au niveau d'un travail contre la nature figée de la langue. Dans ce sens, l'aventure autobiographique de Sarraute constitue une reprise d'une préoccupation perpétuelle que l'on remarque dès la publication de Tropismes. Or, cette lutte contre les clichés et les contraintes se poursuit également par rapport au texte autobiographique, car au lieu de se contenter d'un simple réemprunt des formes traditionnelles du genre, Sarraute adopte une stratégie d'innovation. Tout en réutilisant certains traits typiques de ce genre, Sarraute les retravaille, pour faire ainsi un mouvement en arrière et en avant. À cet égard, Enfance illustre la capacité autobiographique d'être "moins la restitution historique du passé que

32 Nathalie Sarraute, Entre la vie et la mort, op. cit., p. 23.

33 Nathalie Sarraute, Théâtre, Paris, Gallimard, p. 129. Ces mêmes maisons sont également commentées dans Entre la vie et la mort, op. cit., p. 26.

34 Nathalie Sarraute, op. cit., p. 63. 
la construction d'une image du passé pour expliquer le présent et éclairer l'avenir $₫ 35$.

35 Philippe Lejeune, "Peut-on innover en autobiographie?", dans M. Neyraut, J. B. Pontalis et al., L'autobiograpbie, Paris, Les Belles Lettres, 1990, p. 87. 